Article

\title{
Symmetry and Structure in the POT Family of Proton Coupled Peptide Transporters
}

\author{
Simon Newstead \\ Department of Biochemistry, University of Oxford, Oxford OX1 3QU, UK; simon.newstead@bioch.ox.ac.uk \\ Academic Editor: John Vakonakis \\ Received: 17 April 2017; Accepted: 8 June 2017; Published: 14 June 2017
}

\begin{abstract}
The POT family of proton coupled oligopeptide transporters belong to the Major Facilitator Superfamily of secondary active transporters and are found widely distributed in bacterial, plant, fungal and animal genomes. POT transporters use the inwardly directed proton electrochemical gradient to drive the concentrative uptake of di- and tri-peptides across the cell membrane for metabolic assimilation. Mammalian members of the family, PepT1 and PepT2, are responsible for the uptake and retention of dietary protein in the human body, and due to their promiscuity in ligand recognition, play important roles in the pharmacokinetics of drug transport. Recent crystal structures of bacterial and plant members have revealed the overall architecture for this protein family and provided a framework for understanding proton coupled transport within the POT family. An interesting outcome from these studies has been the discovery of symmetrically equivalent structural and functional sites. This review will highlight both the symmetry and asymmetry in structure and function within the POT family and discuss the implications of these considerations in understanding transport and regulation.
\end{abstract}

Keywords: membrane transport; major facilitator superfamily; peptide transport

\section{Introduction}

The uptake and retention of dietary peptides in mammals is carried out by the plasma membrane transporters, PepT1 (SLC15A1) and PepT2 (SLC15A2) [1,2], which are expressed in the cells of the small intestine and kidney respectively. PepT1 and PepT2 are proton-coupled symporters, using the inwardly directed proton electrochemical gradient $\left(\Delta \mu \mathrm{H}^{+}\right)$to drive the concentrative uptake of diand tri-peptides across the plasma membrane [3,4]. PepT1 and PepT2 also recognize and transport a number of important drug families, including beta-lactam antibiotics and anti-cancer agents into the body (reviewed in [5]) and are important targets in the ongoing attempts of the pharmaceutical industry to improve the pharmacokinetic properties of drug molecules $[5,6]$. PepT1 and PepT2 are members of the more widely distributed proton dependent oligopeptide transporter or POT family (TC 2.A.17) which are evolutionarily well conserved from bacteria to man [7]. Consistent with their role in the nutritional assimilation of peptides POT family transporters have evolved remarkably promiscuous binding sites, and are estimated to recognize $>8000$ different di- and tri-peptides ligands [8,9]. In plants the POT family, also known as the NPF or NRT1/PTR family [10], have evolved to recognize a more diverse range of molecules [10]. These include nitrate for metabolism, glucosinolates for seed defence and phytohormones for growth and developmental control [11-13]. In 2014, the crystal structure of the first plant member of the POT family, NRT1.1, was determined [14]. NRT1.1 is a proton coupled nitrate transporter and cannot recognise peptides. However, the structure revealed that much of the proton coupling residues identified in the bacterial and mammalian peptide transporters remained intact. The ability of one family of transporters to recognise such a diverse range of molecules, yet still retain much of the proton coupling machinery is still a biochemical puzzle and very likely at the centre of how this family has achieved such diversity in ligand recognition. 
POT transporters structurally belong to the major facilitator superfamily (MFS) of secondary active transporters. MFS transporters are integral membrane proteins that consist of at least 12 roughly equal transmembrane helices [15]. The $12 \mathrm{TM}$ helices fold into two 6TM bundles, which form a V-shaped molecule in the membrane. Rather uniquely among secondary active transporters these two domains functionally connect only through side chain interactions, without any crossing over of the back-bone chain. Essentially one can think of an MFS transporter as two independent 6TM proteins that have evolved to function together within the membrane. MFS transporters operate via an alternating access mechanism, wherein a central ligand binding site is alternately exposed to either side of the membrane in response to ligand binding [16] (Figure 1A). Recent crystal structures of several bacterial POT family members have revealed conserved structural features that support this model, including identification of the "gating" helices that allow access to a central binding site and location of conserved salt bridge interactions that act as proton binding and release "locks" or salt bridges [17,18], that enable conformational changes to be linked to proton movement. In this article I will outline the structural symmetry within these proteins and highlight the equivalent functional symmetry that has emerged following the biochemical characterisation of these remarkable transport proteins.

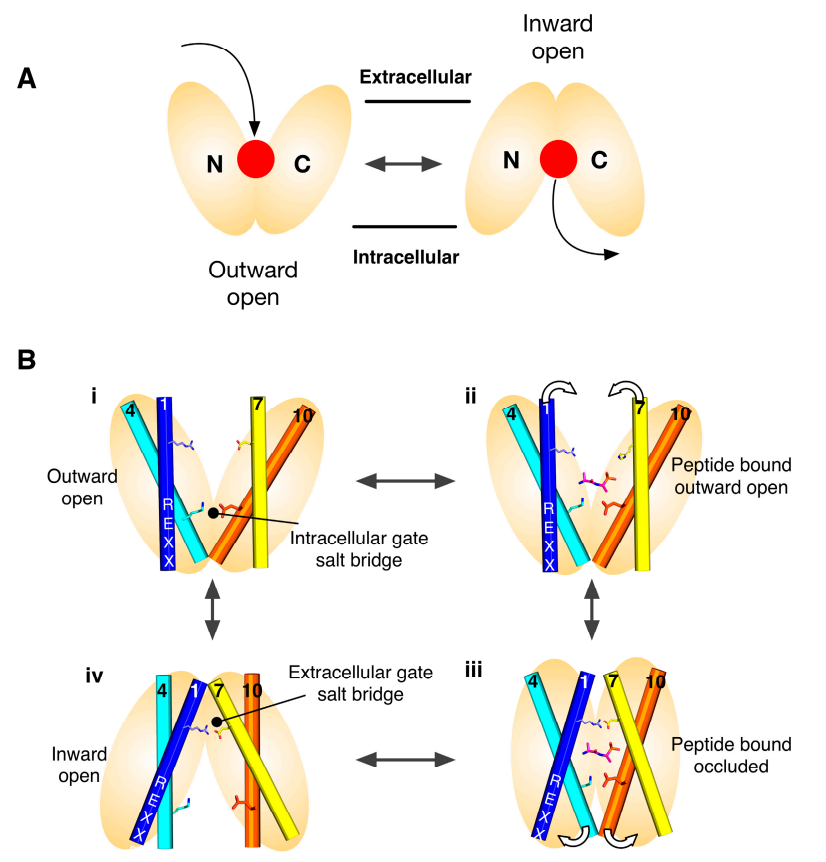

Figure 1. Alternating access model for proton coupled peptide transport. (A) Transport is achieved through the alternating access model, wherein a central ligand binding site, (red dot), is alternately exposed to either side of the membrane. MFS transporters are composed of two six helical bundles that sit in the membrane in a " $\mathrm{V}$ " shaped configuration; (B) Transport is predominantly controlled through the opening and closing of two gates that sit on either side of a central binding site and which are constructed out of the transmembrane helices that make up the two bundles. Specifically, the gates are made helices 1,2,4,5 from the N-terminal bundle and 7,8,10,11 from the C-terminal bundle, which are also the inverted topology repeat units. In many POT family members two salt bridge interactions are seen coordinating the open/closed state of these gates, above and below the peptide binding site respectively. Proton binding/release from conserved proton binding sites act to drive the transport cycle in the forward direction, thereby concentrating peptides inside the cell.

\section{Symmetry-Exchange Underlies the Overall Conformational Change with the Major Facilitator Superfamily}

Secondary active transporters use the energy stored in secondary ion gradients to drive the transport of ligands across the membrane. With respect to the POT family, which use the inwardly 
directed proton electrochemical gradient $\left(\Delta \mu \mathrm{H}^{+}\right)$, this means that proton binding and release within the protein must facilitate the structural changes required to alternate between an outward facing state at the start of the transport cycle, to an inward facing state at the end. Figure 1B shows the current model for proton coupled transport in the POT family. From an outward facing state (i), where the central peptide binding site is open to the outside of the cell, the intracellular gate is closed and stabilised through a salt bridge interact between helices 4 and 10. In this state the central binding site is accessible to the outside of the cell and can bind a peptide and protons. One site of proton binding is the carboxylic group on TM-7. Once bound the transporter is able to close up around the peptide, forming an occluded state (ii), where the proton is able to move from the extracellular gate salt bridge to the intracellular gate salt bridge (iii). In this state, the intracellular gate salt bridge is disrupted, allowing the intracellular gate to open and release peptide and protons in to the cell (iv). The transporter is then able to re-set to the outward facing state through re-formation of the intracellular gate salt bridge (reviewed in [19]).

A key problem when trying to understand how these proteins perform such membrane gymnastics is to have a complete set of structures that reveal the different conformations depicted in Figure 1B. However, membrane proteins are notoriously difficult to crystallise and even more difficult to trap in specific states. To address this challenge the structural community has taken advantage of the internal symmetry displayed within the structures of these proteins and used this information to model different states of the transport cycle with remarkable success [20].

The MFS is the largest superfamily of secondary active transporters, containing over 70 different families [21]. Structures of members belonging to several MFS families reveal a common fold consisting of two bundles of six TM $\alpha$ helices that come together to form a " $\mathrm{V}$ "-shaped transporter with a central substrate binding site (Figures 1A and 2B) [22,23]. Intriguingly each 6-TM bundle can be further divided into two so-called inverted-topology repeat units consisting of 3-TM each (giving four 3-TM repeats in total, labelled A-D in Figure 2A) [24,25]. Overall therefore the MFS reveals both a twofold symmetry axis running parallel to the membrane, relating each 6-TM bundle to one another, and two further twofold symmetry axes running normal to the membrane, relating each 3-TM inverted topology repeat. The significance of this internal symmetry in understanding how these proteins work was elegantly demonstrated when the outward open conformation of a structurally related MFS transporter, LacY, the E. coli lactose permease, was built using the crystal structure of the inward open protein [26]. Essentially, the authors showed that through careful sequence alignment and structure threading, the sequence of each 3-TM inverted topology repeat unit could be transposed onto the symmetrically equivalent unit, which in the case of the MFS turns out to be A-C and B-D. The subsequent outward open state, which had previously been recalcitrant to crystallisation, was subsequently determined and shown to agree, at least qualitatively, with the "repeat-swapped" model [27]. The conceptual advance in this work was the realisation that a "symmetry-exchange" mechanism underlies the overall conformational change with the MFS and has been shown, more broadly, to operate within all currently determined secondary active transporter families [20]. More specifically, that for these proteins to move between the outward and inward facing states (Figure 1A), all this is required is for the inverted topology repeats to adopt their symmetrically equivalent positions. This was a significant breakthrough in our understanding of the energetic landscape within which secondary active transporters operate [28]. The symmetry exchange model suggests that the outward facing and inwards states are energetically close together, with only a small energy barrier separating them. However, the actual energy landscape of a proton coupled MFS symporter has not been determined at the single molecule level in an energised membrane system. It is likely that specific high energy kinetic barriers do exist that regulate the transport cycle and ultimately result in concentrative ligand accumulation inside the cell. A relatively flat energy landscape however may explain in part how external ion and ligand gradients are able to shift the conformational equilibrium between outward and inward facing states, even though the binding of a proton and ligand may only provide a few $\mathrm{kcal}^{\mathrm{mol}}{ }^{-1}$ in energy. However, for POT family transporters at least, this is unlikely to be the full story 
given that all current crystal structures have been captured in the inward facing conformation [29]. Interestingly, this observation suggests an apparent asymmetry with respect to the energetics of the transport cycle that favours the inward facing over the outward facing state.

A
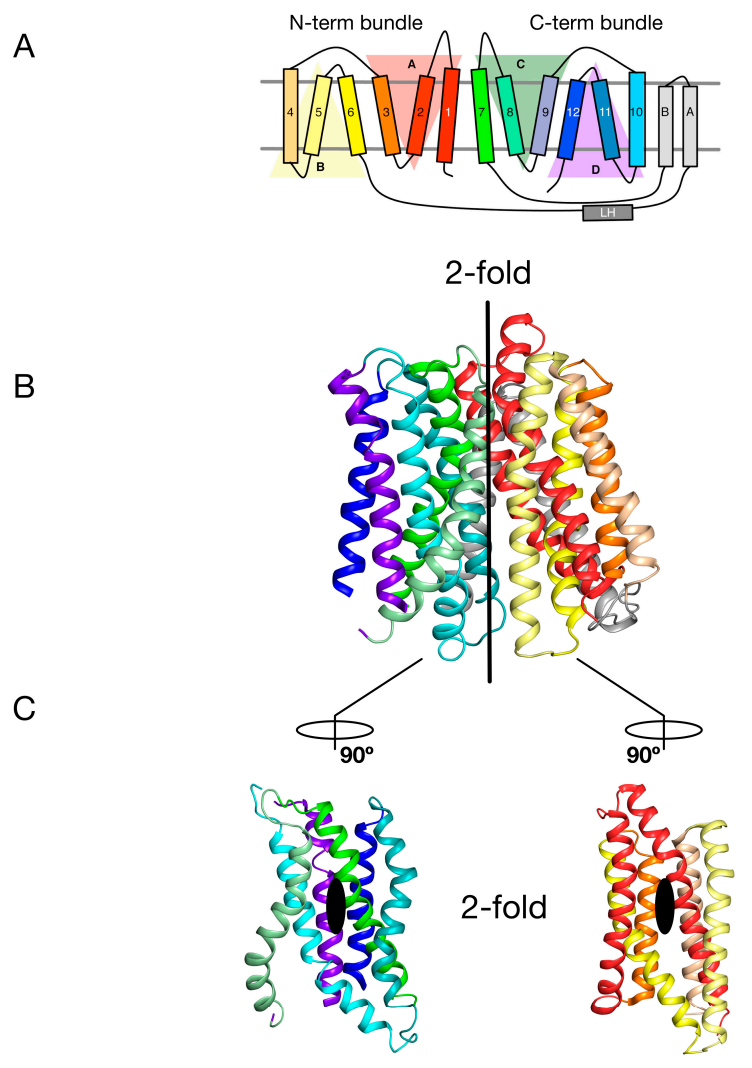

Figure 2. Internal symmetry within the POT family. (A) Proton coupled oligopeptide transporters comprise of four inverted topology repeats, two within each of the N- and C-terminal bundles. Each repeat unit (labelled A-D) is itself made up of three transmembrane alpha helices (labelled 1-12). Many bacterial POT family members contain an additional two transmembrane alpha helices (labelled A \& B), which are inserted between the canonical N-terminal bundle (1-6) and C-terminal bundle (7-12); (B) Crystal structure of PepT $\mathrm{T}_{\mathrm{st}}$ (PDB: 4APS) coloured as in the topology diagram in A. The vertical bar delineates the 2-fold symmetry axis that relates the N-terminal bundle onto the C-terminal bundle; (C) Open book view of the transporter, showing the second set of 2-fold axes, which relate each 3-TM inverted topology repeat within the respective $\mathrm{N}$ - and C-terminal bundles. Image adapted from [27].

\section{Gating Topology with the POT Family}

Recently we used the repeat swapped methodology to propose a 'gating' topology for how POT transporters move between inward and outward facing states in response to proton and peptide binding [27]. To occlude the peptide binding site to either side of the membrane, POT transporters form gates, which are transient constrictions formed by the close packing of several transmembrane helices. These gates block entry or exit to the central cavity and are stabilized by interactions between transmembrane helices, specifically through key salt bridges, which in turn are controlled by peptide binding and release. The structures of two POT transporters from bacteria, $\mathrm{PepT}_{\mathrm{So}}$ and $\mathrm{PepT}_{\mathrm{St}}$ showed that the periplasmic gate is formed by helices $\mathrm{H} 1$ \& $\mathrm{H} 2$ packing against $\mathrm{H} 7$ \& $\mathrm{H} 8[17,18]$. The structure of PepT $\mathrm{T}_{\mathrm{So}}$ was captured in an asymmetric inward-occluded conformation and its proposed cytoplasmic gate is sufficiently narrow that, although the binding site is contiguous with the intracellular medium, peptides are likely to be sterically impeded from exit [30]. In contrast, the structure of $\operatorname{Pep}_{\mathrm{St}}$ is more symmetric and, as a result, peptides are not prevented from exiting by the cytoplasmic gate. 
Comparing the structures of PepT $\mathrm{So}_{\mathrm{So}}$ and $\mathrm{PepT}_{\mathrm{St}}$ in more detail revealed that helices H7, H10, and H11 in the C-terminal bundle of PepT $\mathrm{So}_{\mathrm{S}}$ had shifted relative to the inward-open conformation of PepT $\mathrm{T}_{\mathrm{St}}$. This asymmetry in the motion of the transmembrane $\alpha$ helices suggested that a dynamic movement of helices within the two six-helical bundles may be required for the central cavity to be alternately exposed to both sides of the membrane, as observed in LacY [31], the archetypal proton coupled MFS transporter.

Through an analysis of the inverted topology repeats of the POT family a repeat swapped model of the outward facing state of PepT $\mathrm{So}_{\mathrm{S}}$ was recently built (Figure 3A) [27]. This model was experimentally verified using site directed spin labelling and double electron-electron resonance (DEER) spectroscopy, which measures the distance between two nitroxide spin labels attached to different helices within the protein [32]. In building this model and analysing the DEER distance distributions we noticed that within each of the 3-TM inverted topology repeats the first two helices form the mobile gates that alternatively open and close during the transport cycle. In contrast, the third helix in each repeat $\mathrm{H} 3$, $\mathrm{H6}, \mathrm{H9}, \mathrm{H12}$, sit on the outside of the transporter and we propose create a stable platform around which the eight internal helices move against (Figure 3B). We termed this the "gating topology" to emphasise the difference between structural topology, i.e., how the TM helices are arranged with respect to one another and the membrane, versus the functional role that these helices play in the transport cycle.

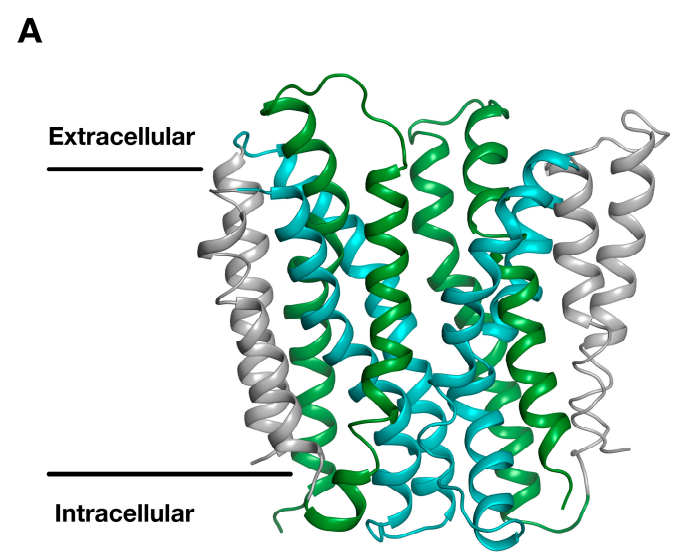

B

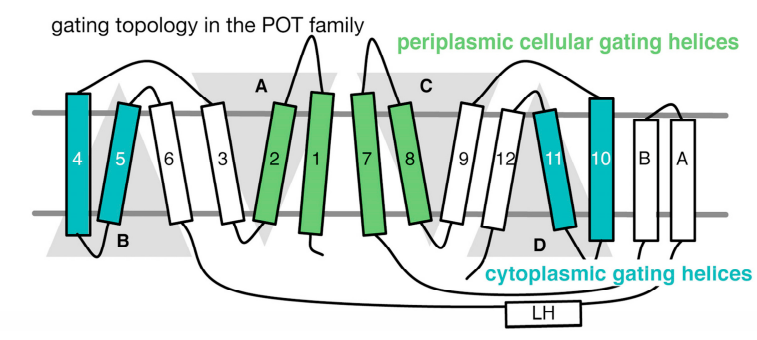

Figure 3. The periplasmic and cytoplasmic gates are formed from two bundles of four $\alpha$ helices. (A) An outward open model for the POT transporter PepTSt was recently built using the symmetry present within the inverted topology repeats (Samsudin); (B) The first two helices from each repeat unit therefore contribute to either the periplasmic gate (green) or the cytoplasmic gate (cyan). The third helix in each repeat-swapped unit is located at the periphery, and does not play a direct role in gating the transport. Image adapted from [27]. 


\section{Salt Bridge Networks and Proton Binding}

Following our identification of the "gating" helices, we then pursued several lines of evidence to identify and understand how these helices where functionally cooperating to open and close in response to proton and peptide binding and release. Using several crystal structures of POT family transporters from Shewanella oniedensis $\left(\mathrm{Pep}_{\mathrm{So}}\right)$, Streptococcus thermophilus $\left(\mathrm{Pep}_{\mathrm{St}}\right)$ and Geobacillus kaustophilus (GkPOT) combined with in vitro liposome based assays, we were able to identify the main sites of proton binding $[17,18]$. These were shown to be two conserved salt bridge interactions between the two sets of gate helices, positioned above and below the main peptide binding site (Figure 1B). The most conserved of these salt bridge interactions involves a lysine on $\mathrm{H} 4$ that is predicted to interact with a glutamate on H10, stabilising the closed state of the intracellular gate. This interaction is found in all POT family transporters to date and also in the distantly related plant nitrate transporter, NRT1.1 [14]. On the extracellular gate we see a symmetrically equivalent interaction between $\mathrm{H} 1$ and H7, however, this interaction is less conserved. In PepT $\mathrm{T}_{\mathrm{t}}$ and GkPOT this interaction is made by an arginine on $\mathrm{H} 1$ and a conserved glutamate on $\mathrm{H} 7$, whereas in PepT $\mathrm{T}_{\mathrm{So}}$, and very likely the mammalian PepT1 and PepT2 transporters, the interaction is through a conserved histidine on $\mathrm{H} 2$ to an aspartate on $\mathrm{H} 7$ [30]. Interestingly, this interaction is absent in $\mathrm{PepT}_{\mathrm{So} 2}$, a related transporter from S. oniedensis [33] and also NRT1.1, showing that this region of the transporter is able to change. A further site of proton binding was also discovered in the POT family, residing in the ExxER motif on H1, with a key role in transport [18]. The position of the arginine in this motif close to the lysine on $\mathrm{H} 4$ suggests this motif plays an important role in regulating the protonation state, or $\mathrm{pKa}$, of the lysine during transport or in response to peptide binding. As discussed below, this is an example of functional asymmetry within the POT family.

\section{Peptide Binding and the Role of Specificity Pockets in Ligand Recognition}

Insights into the biochemical basis for peptide binding and selectivity have recently come from three different POT family members crystallised in complex with peptides [17,34,35]. Detailed analyses of the binding interactions in these structures have been published recently $[19,29]$ and will not be discussed in detail here. However, it is worth considering the findings of these studies in the context of symmetry within these systems. PepT $\mathrm{T}_{\mathrm{St}}$ is the most recently crystallised peptide transporter captured in complex with the physiological peptides L-ala-L-phe (AF) and L-ala-L-ala-L-ala (AAA) (Figure 4A). Through comparisons of structures of GkPOT with the antibacterial peptide alaphosfalin and PepTSo2 with peptides AY, AYA and AAA, several specificity pockets are evident (Figure 4B). There is a polar pocket, formed from side chains contributed from TM's 1, 4, 5 and 8 that sits next to the ExxERF pocket on TM1 and 4. These two regions are opposite an acidic patch on TM7 and 10 in PepT $\mathrm{St}_{\mathrm{St}}$ and a hydrophobic pocket, constructed of aromatic side chains from TM's 2, 11. Several lines of biochemical evidence suggest that ligand selectivity is the result of interactions between the side chains of the peptide and these pockets [34-36], which in turn promote the conformational changes required for transport. It is unclear what role, if any, symmetry plays in peptide recognition. The orientation of the pockets within the transporter suggests each 6-TM bundle plays a role in peptide recognition. However, several lines of evidence suggest that asymmetry during transport, with the C-terminal bundle being more dynamic that the N-terminal bundle. For example, the binding of the AF peptide to $\mathrm{PepT}_{\mathrm{St}}$ promotes the formation of the hydrophobic pocket, which in turn brings TM10 and 11 closer to TM4, 5, closing the intracellular gate (Figure 4C) [18] and the semi-occluded structure of PepT $\mathrm{So}_{\mathrm{S}}$ shows considerable movement in only the C-terminal helices compared to LacY, the archetypical MFS transporter [37]. We must however await the outward facing state of a POT family member to see whether symmetrically equivalent movements occur in TM1,2 or whether asymmetry in the helices is a hallmark of transport cycle in the POT family.

It is also worth noting that within the binding site at least two regions of proton binding have been identified. These are the acidic patch, namely the Asp or Glu side chain on TM7 and 10 [17] and the ExxERF motif on TM1 and 4 [18]. It is interesting, in the context of this review, to consider that TM1 
and 7 are the first helices in the inverted topology repeat that control the periplasmic gate, and TM4 and 10 form part of the intracellular gate (Figure 3B), but that the side chains that constitute these pockets come from both sets of helices. It is likely that this enables the transporter to couple peptide transport to proton binding and release on either side of the membrane. What is currently not clear is the role played by protons in peptide binding. Are they required for high affinity binding, or merely to drive transport? We will need to dissect out this question to fully understand how these proteins have evolved and understand the mechanistic differences and similarities between the family members.
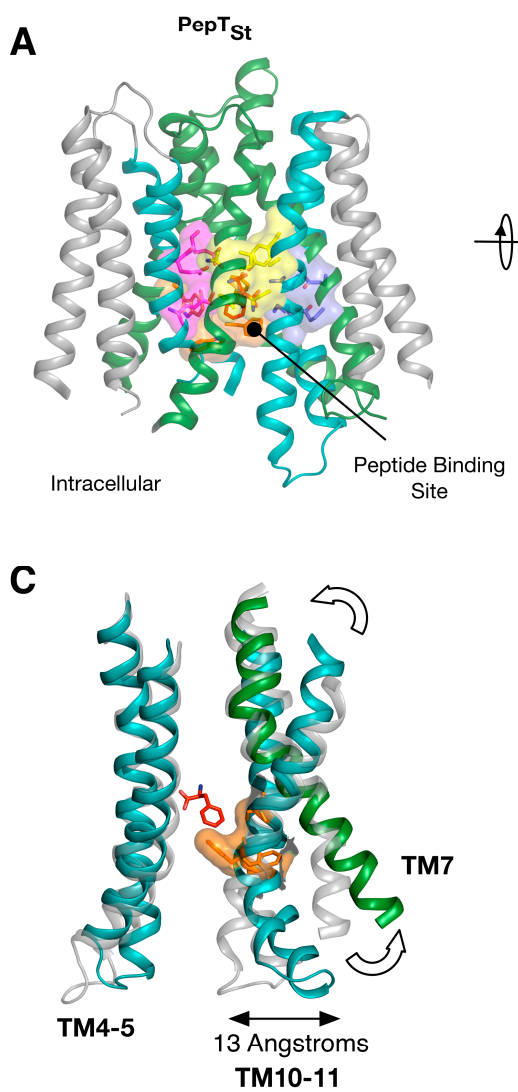

B

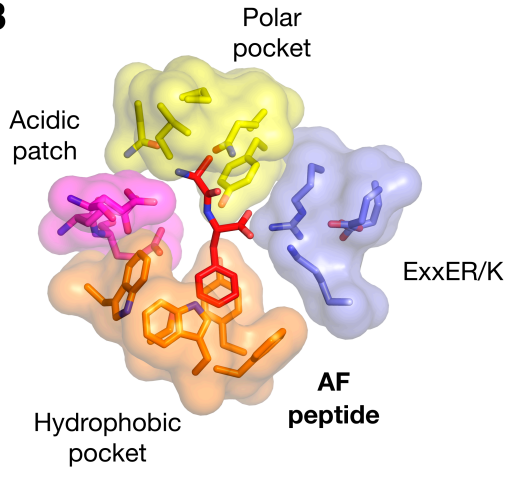

Figure 4. Equivalent sites in evolutionarily distant proteins have similar effects on function. (A) Crystal structure of PepT $T_{S t}$ shown in the plane of the membrane with the di-peptide Ala-Phe (PDB: 4D2C) (red) shown as sticks. The structure has been coloured according to Figure 3; (B) Close up view of the specificity pockets formed around the Ala-Phe peptide; (C) Overlay of the inward open (PDB: 4APS) and $\mathrm{AF}$ bound structures of $\mathrm{PepT}_{\mathrm{St}}$, highlighting the asymmetry in moving from the inward open to the occluded state upon peptide binding. Images adapted from [18,19].

\section{Regulation and Structural Adaptation}

Although recent structural and functional work on the POT family has focused on mapping out the key steps in the transport cycle and understanding the biochemistry of the binding site, the regulation of these transporters in the context of the cell has recently emerged as an important component in the biology and evolutionary adaption of these proteins. The Arabidopsis thaliana nitrate transporter, NRT1.1 (NPF6.3), is the founding member [10] of the NRT1/PTR family of nitrate/peptide transporters in plants and shares significant sequence identity to mammalian and bacterial POT peptide transporters $[10,14]$. NRT1.1 is a proton coupled nitrate transporter expressed in the root hair cells of plants and functions to transport nitrate from the environment for metabolic assimilation. Unlike other POT family transporters however, NRT1.1 exhibits a biphasic transport profile with two $K_{\mathrm{M}}$ values for its substrate, nitrate [38]. In conditions of high nitrate availability ( $\left.>1 \mathrm{mM}\right)$ NRT1.1 behaves as a low-affinity, high capacity transporter $\left(K_{\mathrm{M}} \approx 4 \mathrm{mM}\right)$. This enables the plant to take up 
as much nitrate as possible from the soil when nitrate is available. However, when nitrate levels fall below $1 \mathrm{mM}$, for example, following heavy rain, NRT1.1 is switched into a high-affinity transporter $\left(K_{\mathrm{M}} \approx 40 \mu \mathrm{M}\right)$, enabling the plant to quickly transport the disappearing nutrient.

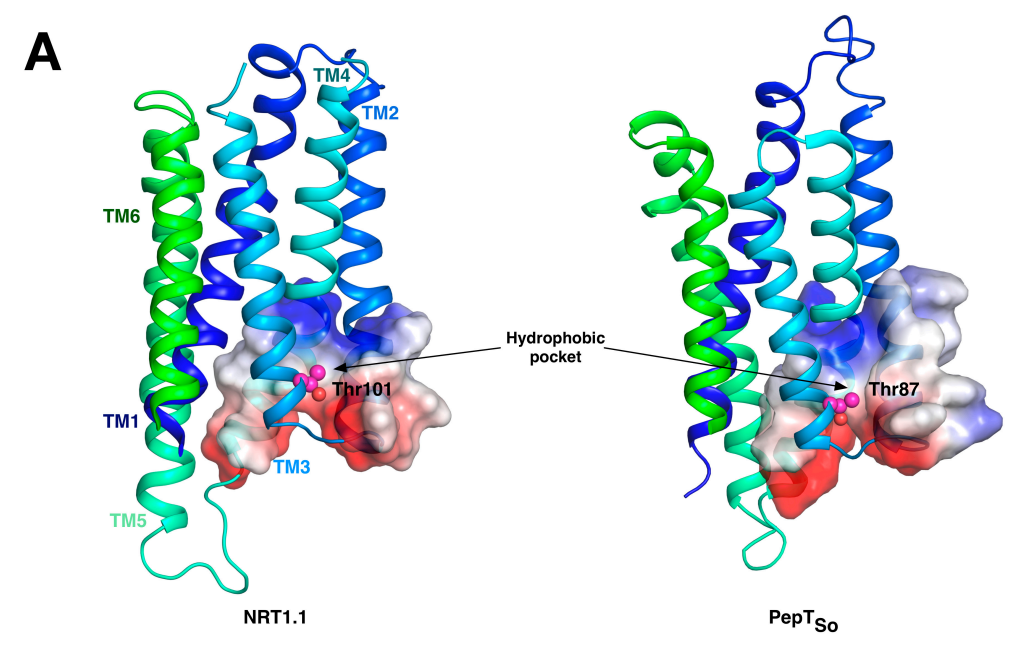

B

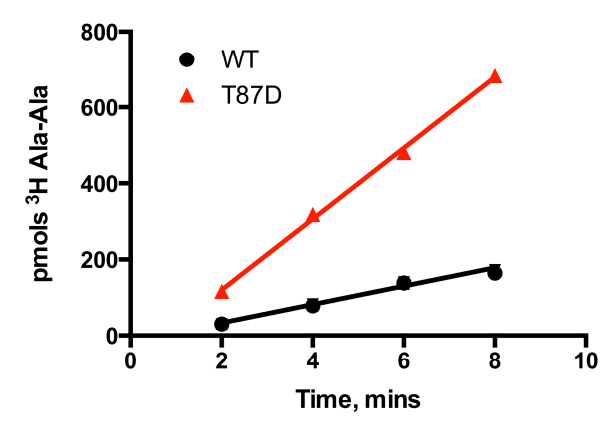

Figure 5. Equivalent sites in evolutionarily distant proteins have similar effects on function. (A) The N-terminal domains of the plant NRT1.1 and the bacterial peptide transporters are very similar. The location of the phosphorylation site in NRT1.1 (Thr101) and the equivalent threonine in $\mathrm{PepT}_{\text {So }}$ (Thr87) are highlighted as magenta spheres and located in similar hydrophobic pockets; (B) The Thr87Asp variant of PepT $\mathrm{T}_{\mathrm{So}}$ showed a fourfold increase in the rate of peptide transport in liposome based assays, in a similar manner observed for the plant transporter NRT1.1. Images adapted from [14].

This switch occurs following phosphorylation of an intracellular threonine, Thr 101, by the kinase CIPK23 [39]. This regulatory mechanism allows for the rapid adaption to changing nitrate levels before the dedicated high affinity NRT2 transporter family is expressed [40]. These observations suggested a complex interplay between transporter activity and post-translational modification at the molecular level. We and others recently determined the molecular basis for this kinetic switch, which depends on the ability of the phosphorylation to induce structural instability within the protein [14,41]. Specifically, the crystal structure of NRT1.1 revealed that Thr101 was located on H3, within the first 3-TM repeat, with the side chain of the amino acids pointing into a conserved hydrophobic pocket (Figure 5A). We showed that mutating the Thr to an aspartate, which functionally mimics the phosphorylated state, results in an increase of the transport rate. Our explanation for this effect was that the negative charge on the aspartate would destabilise the N-terminal 6-TM bundle, in effect disrupting the packing between the two 3-TM inverted topology repeats. We reasoned that this increase in structural flexibility would enable the transporter to more quickly move through the transport cycle, reducing the rate determining step and shifting the energetic landscape of the transport cycle. This effect would explain 
the lowering of the $\mathrm{K}_{\mathrm{M}}$ values observed in the cell based uptake assays. We also observed an equally intriguing observation. When we made the equivalent mutation in the bacterial POT family transporter PepT $_{\text {So }}$, Thr87Asp, we observed the same effect (Figure 5B). This result demonstrated a functional symmetry in the rate determining step between the bacterial peptide transporters and plant nitrate transporter, NRT1.1 that we are currently studying further.

\section{Conclusions}

One of the major outcomes from the structural studies on secondary active transporters in recent years has been the importance of symmetry [20,42]. It is now clear with each new structural fold that is reported that secondary active transporters have been built around the thermodynamic principle of energetically equivalent states, which can be shifted in response to ligand binding. The POT family exhibit several unique characteristics with respect to symmetry, not least the general architecture of the MFS fold, but also the functional symmetry in the location of the salt bridge interactions between the extracellular and intracellular gates. However, asymmetry also plays an important role. Early on in the structural studies of the MFS it was proposed that alternating access transport would be achieved through the rigid body rocking of the N-terminal 6-TM bundle against the C-terminal 6-TM bundle, with the ligand and protons binding in the middle [43]. In the POT family the structure of PepT $\mathrm{So}_{\mathrm{So}}$ in the asymmetric occluded state suggested this two state model was likely too simple, and that in fact transport is achieved through a far more complicated choreography of helical movement in response to proton and peptide binding [23,29]. Our experiments, models, and molecular dynamics simulations suggest that in the POT family, the first six helices are less dynamic than the last six, consistent with a functional separation between the $\mathrm{N}$ - and C-terminal bundles. A similar asymmetry was also observed in LacY [44], suggesting this separation may be a feature of proton coupled MFS members. We also observe a breakdown in the simple two salt bridge mechanism with respect to $\mathrm{PepT}_{\mathrm{So} 2}$ and NRT1.1, which clearly don't require a second salt bridge to stabilise the close state of the extracellular gate. Symmetry however has clearly played an important role in helping to build conceptual models for how POT family transporters work and will continue to provide further insight into the mechanism of proton coupled transport across the membrane.

Acknowledgments: This work was funded by Wellcome through an Investigator Award to SN (102890/Z/13/Z).

Conflicts of Interest: The author declares no conflict of interest with respect to this work.

\section{References}

1. Adibi, S.A. The oligopeptide transporter (Pept-1) in human intestine: Biology and function. Gastroenterology 1997, 113, 332-340. [CrossRef]

2. Matthews, D.M. Protein Absorption: Development and Present State of the Subject; Wiley-Liss: New York, NY, USA, 1991; p. 414.

3. Fei, Y.J.; Kanai, Y.; Nussberger, S.; Ganapathy, V.; Leibach, F.H.; Romero, M.F.; Singh, S.K.; Boron, W.F.; Hediger, M.A. Expression cloning of a mammalian proton-coupled oligopeptide transporter. Nature 1994, 368, 563-566. [CrossRef] [PubMed]

4. Daniel, H.; Rubio-Aliaga, I. An update on renal peptide transporters. Am. J. Physiol. Ren. Physiol. 2003, 284, F885-F892. [CrossRef] [PubMed]

5. Brandsch, M. Drug transport via the intestinal peptide transporter PepT1. Curr. Opin. Pharmacol. 2013, 13, 881-887. [CrossRef] [PubMed]

6. Smith, D.E.; Clemencon, B.; Hediger, M.A. Proton-coupled oligopeptide transporter family SLC15: Physiological, pharmacological and pathological implications. Mol. Asp. Med. 2013, 34, 323-336. [CrossRef] [PubMed]

7. Daniel, H.; Spanier, B.; Kottra, G.; Weitz, D. From bacteria to man: Archaic proton-dependent peptide transporters at work. Physiology 2006, 21, 93-102. [CrossRef] [PubMed] 
8. Ito, K.; Hikida, A.; Kawai, S.; Lan, V.T.; Motoyama, T.; Kitagawa, S.; Yoshikawa, Y.; Kato, R.; Kawarasaki, Y. Analysing the substrate multispecificity of a proton-coupled oligopeptide transporter using a dipeptide library. Nat. Commun. 2013, 4, 2502. [CrossRef] [PubMed]

9. Biegel, A.; Knutter, I.; Hartrodt, B.; Gebauer, S.; Theis, S.; Luckner, P.; Kottra, G.; Rastetter, M.; Zebisch, K.; Thondorf, I.; et al. The renal type $\mathrm{H}^{+}$/ peptide symporter PEPT2: Structure-affinity relationships. Amino Acids 2006, 31, 137-156. [CrossRef] [PubMed]

10. Léran, S.; Varala, K.; Boyer, J.-C.; Chiurazzi, M.; Crawford, N.; Daniel-Vedele, F.; David, L.; Dickstein, R.; Fernandez, E.; Forde, B.; et al. A unified nomenclature of nitrate transporter 1/peptide transporter family members in plants. Trends Plant Sci. 2014, 19, 5-9. [CrossRef] [PubMed]

11. Tsay, Y.-F.; Chiu, C.-C.; Tsai, C.-B.; Ho, C.-H.; Hsu, P.-K. Nitrate transporters and peptide transporters. FEBS Lett. 2007, 581, 2290-2300. [CrossRef] [PubMed]

12. Boursiac, Y.; Léran, S.; Corratgé-Faillie, C.; Gojon, A.; Krouk, G.; Lacombe, B. ABA transport and transporters. Trends Plant Sci. 2013, 18, 325-333. [CrossRef] [PubMed]

13. Nour-Eldin, H.H.; Andersen, T.G.; Burow, M.; Madsen, S.R. NRT/PTR transporters are essential for translocation of glucosinolate defence compounds to seeds. Nature 2012, 488, 531-534. [CrossRef] [PubMed]

14. Parker, J.L.; Newstead, S. Molecular basis of nitrate uptake by the plant nitrate transporter NRT1.1. Nature 2014, 507, 68-72. [CrossRef] [PubMed]

15. Abramson, J.; Iwata, S.; Kaback, H.R. Lactose permease as a paradigm for membrane transport proteins (review). Mol. Membr. Biol. 2004, 21, 227-236. [CrossRef] [PubMed]

16. Jardetzky, O. Simple allosteric model for membrane pumps. Nature 1966, 211, 969-970. [CrossRef] [PubMed]

17. Doki, S.; Kato, H.E.; Solcan, N.; Iwaki, M.; Koyama, M.; Hattori, M.; Iwase, N.; Tsukazaki, T.; Sugita, Y.; Kandori, H.; et al. Structural basis for dynamic mechanism of proton-coupled symport by the peptide transporter pot. Proc. Natl. Acad. Sci. USA 2013, 110, 11343-11348. [CrossRef] [PubMed]

18. Solcan, N.; Kwok, J.; Fowler, P.W.; Cameron, A.D.; Drew, D.; Iwata, S.; Newstead, S. Alternating access mechanism in the pot family of oligopeptide transporters. EMBO J. 2012, 31, 3411-3421. [CrossRef] [PubMed]

19. Newstead, S. Recent advances in understanding proton coupled peptide transport via the pot family. Curr. Opin. Struct. Biol. 2016, 45, 17-24. [CrossRef] [PubMed]

20. Forrest, L.R. Structural symmetry in membrane proteins. Annu. Rev. Biophys. 2015, 44, 311-337. [CrossRef] [PubMed]

21. Reddy, V.S.; Shlykov, M.A.; Castillo, R.; Sun, E.I.; Saier, M.H. The major facilitator superfamily (MFS) revisited. FEBS J. 2012, 279, 2022-2035. [CrossRef] [PubMed]

22. Drew, D.; Boudker, O. Shared molecular mechanisms of membrane transporters. Annu. Rev. Biochem. 2016, 85, 543-572. [CrossRef] [PubMed]

23. Law, C.J.; Maloney, P.C.; Wang, D.-N. Ins and outs of major facilitator superfamily antiporters. Annu. Rev. Microbiol. 2008, 62, 289-305. [CrossRef] [PubMed]

24. Madej, M.G.; Dang, S.; Yan, N.; Kaback, H.R. Evolutionary mix-and-match with mfs transporters. Proc. Natl. Acad. Sci. USA 2013, 110, 5870-5874. [CrossRef] [PubMed]

25. Madej, M.G.; Kaback, H.R. Evolutionary mix-and-match with MFS transporters II. Proc. Natl. Acad. Sci. USA 2013, 110, E4831-E4838. [CrossRef] [PubMed]

26. Radestock, S.; Forrest, L.R. The alternating-access mechanism of MFS transporters arises from inverted-topology repeats. J. Mol. Biol. 2011, 407, 698-715. [CrossRef] [PubMed]

27. Fowler, P.W.; Orwick-Rydmark, M.; Radestock, S.; Solcan, N.; Dijkman, P.M.; Lyons, J.A.; Kwok, J.; Caffrey, M.; Watts, A.; Forrest, L.R.; et al. Gating topology of the proton-coupled oligopeptide symporters. Structure 2015, 23, 290-301. [CrossRef] [PubMed]

28. Forrest, L.R.; Krämer, R.; Ziegler, C. The structural basis of secondary active transport mechanisms. Biochim. Biophys. Acta 2011, 1807, 167-188. [CrossRef] [PubMed]

29. Newstead, S. Molecular insights into proton coupled peptide transport in the PTR family of oligopeptide transporters. Biochim. Biophys. Acta 2015, 1850, 488-499. [CrossRef] [PubMed]

30. Newstead, S.; Drew, D.; Cameron, A.D.; Postis, V.L.G.; Xia, X.; Fowler, P.W.; Ingram, J.C.; Carpenter, E.P.; Sansom, M.S.P.; Mcpherson, M.J.; et al. Crystal structure of a prokaryotic homologue of the mammalian oligopeptide-proton symporters, PepT1 and PepT2. EMBO J. 2011, 30, 417-426. [CrossRef] [PubMed] 
31. Zhou, Y.; Madej, M.G.; Guan, L.; Nie, Y.; Kaback, H.R. An early event in the transport mechanism of LacY protein: INTERACTION BETWEEN HELICES V AND I. J. Biol. Chem. 2011, 286, 30415-30422. [CrossRef] [PubMed]

32. Reginsson, G.W.; Schiemann, O. Pulsed electron-electron double resonance: Beyond nanometre distance measurements on biomacromolecules. Biochem. J. 2011, 434, 353-363. [CrossRef] [PubMed]

33. Guettou, F.; Quistgaard, E.M.; Trésaugues, L.; Moberg, P.; Jegerschöld, C.; Zhu, L.; Jong, A.J.O.; Nordlund, P.; Löw, C. Structural insights into substrate recognition in proton-dependent oligopeptide transporters. EMBO Rep. 2013, 14, 804-810. [CrossRef] [PubMed]

34. Lyons, J.A.; Parker, J.L.; Solcan, N.; Brinth, A.; Li, D.; Shah, S.T.; Caffrey, M.; Newstead, S. Structural basis for polyspecificity in the pot family of proton-coupled oligopeptide transporters. EMBO Rep. 2014, 15, 886-893. [CrossRef] [PubMed]

35. Guettou, F.; Quistgaard, E.M.; Raba, M.; Moberg, P.; Low, C.; Nordlund, P. Selectivity mechanism of a bacterial homolog of the human drug-peptide transporters PepT1 and PepT2. Nat. Struct. Mol. Biol. 2014, 21, 728-731. [CrossRef] [PubMed]

36. Samsudin, F.; Parker, J.L.; Sansom, M.S.; Newstead, S.; Fowler, P.W. Accurate prediction of ligand affinities for a proton-dependent oligopeptide transporter. Cell Chem. Biol. 2016, 23, 299-309. [CrossRef] [PubMed]

37. Newstead, S. Towards a structural understanding of drug and peptide transport within the proton-dependent oligopeptide transporter (POT) family. Biochem. Soc. Trans. 2011, 39, 1353-1358. [CrossRef] [PubMed]

38. Liu, K.H.; Huang, C.Y.; Tsay, Y.F. Chl1 is a dual-affinity nitrate transporter of arabidopsis involved in multiple phases of nitrate uptake. Plant Cell 1999, 11, 865-874. [CrossRef] [PubMed]

39. Ho, C.-H.; Lin, S.-H.; Hu, H.-C.; Tsay, Y.-F. Chl1 functions as a nitrate sensor in plants. Cell 2009, 138, 1184-1194. [CrossRef] [PubMed]

40. Wang, Y.Y.; Hsu, P.K.; Tsay, Y.F. Uptake, allocation and signaling of nitrate. Trends Plant Sci. 2012, 17, 458-467. [CrossRef] [PubMed]

41. Sun, J.; Bankston, J.R.; Payandeh, J.; Hinds, T.R.; Zagotta, W.N.; Zheng, N. Crystal structure of the plant dual-affinity nitrate transporter NRT1.1. Nature 2014, 507, 73-77. [CrossRef] [PubMed]

42. Forrest, L.R. Structural biology. (Pseudo-)symmetrical transport. Science 2013, 339, 399-401. [CrossRef] [PubMed]

43. Abramson, J.; Smirnova, I.; Kasho, V.; Verner, G.; Kaback, H.R.; Iwata, S. Structure and mechanism of the lactose permease of escherichia coli. Science 2003, 301, 610-615. [CrossRef] [PubMed]

44. Serdiuk, T.; Madej, M.G.; Sugihara, J.; Kawamura, S.; Mari, S.A.; Kaback, H.R.; Muller, D.J. Substrate-induced changes in the structural properties of LacY. Proc. Natl. Acad. Sci. USA 2014, 111, E1571-E1580. [CrossRef] [PubMed]

(C) 2017 by the author. Licensee MDPI, Basel, Switzerland. This article is an open access article distributed under the terms and conditions of the Creative Commons Attribution (CC BY) license (http://creativecommons.org/licenses/by/4.0/). 